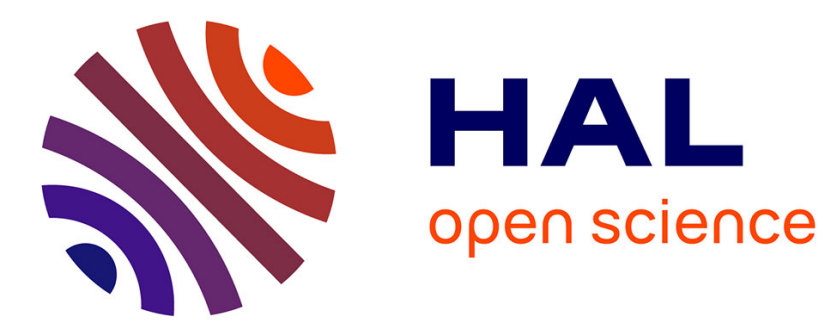

\title{
DDE Transposon as Public Goods
}

Louis Tsakou-Ngouafo, Célia Vicari, Laura Helou, Vivek Keshri, Sabyasachi

Das, Yves Bigot, Pierre Pontarotti

\section{To cite this version:}

Louis Tsakou-Ngouafo, Célia Vicari, Laura Helou, Vivek Keshri, Sabyasachi Das, et al.. DDE Transposon as Public Goods. Evolutionary Biology-A Transdisciplinary Approach, Springer International Publishing, pp.337 - 357, 2020, 10.1007/978-3-030-57246-4_14 . hal-03043351

\section{HAL Id: hal-03043351 \\ https://hal.science/hal-03043351}

Submitted on 7 Dec 2020

HAL is a multi-disciplinary open access archive for the deposit and dissemination of scientific research documents, whether they are published or not. The documents may come from teaching and research institutions in France or abroad, or from public or private research centers.
L'archive ouverte pluridisciplinaire HAL, est destinée au dépôt et à la diffusion de documents scientifiques de niveau recherche, publiés ou non, émanant des établissements d'enseignement et de recherche français ou étrangers, des laboratoires publics ou privés. 


\title{
Chapter 14 DDE Transposon as Public Goods
}

\author{
Louis Tsakou, Célia Vicari, Laura Helou, Vivek Keshri, Sabyasachi Das, \\ Yves Bigot, and Pierre Pontarotti
}

\begin{abstract}
DDE Transposons have been recruited at least four times as site-specific recombination activating gene allowing programmed DNA elimination in eukaryotes. The described cases are RAG in jawed vertebrates, Kat 1 and Alpha 3 in the Kluyveromyces lactis yeast and Piggymac/TPB1 TPB2 and TPB6 in ciliates. The domesticated RAG is the most known case. It constitutes the enzymatic core of the Jawed vertebrates $\mathrm{V}(\mathrm{D}) \mathrm{J}$ recombination machinery. It directs random assembly and joining of gene segments during the development of B and T cells helping in the generation of the enormous gene diversity encoding antibodies or $\mathrm{T}$ cell receptors. It was shown in the case of RAG that the shift from DDE transposon to site-specific recombination activating gene is an evolutionary phenomenon that did not require dramatic changes. This explains why the co-option of DDE transposon as site-specific recombination activating gene can occur in a convergent manner. As numerous genes coding for DDE transposases are widespread through numerous members of the life tree, it is expected that several of them might correspond to domesticated transposons involved in programmed DNA elimination and maybe in the generation of receptor diversity. The domestication of DDE transposon could have been and still be of an extreme importance for organisms' evolution.
\end{abstract}

L. Tsakou $\cdot$ C. Vicari $\cdot$ V. Keshri $\cdot$ P. Pontarotti $(\bowtie)$

Aix Marseille Univ IRD, APHM, MEPHI, IHU Méditerranée Infection, Marseille, France e-mail: Pierre.pontarotti@univ-amu.fr

L. Helou · Y. Bigot

UMR INRAE 0085, CNRS 7247, PRC, Centre INRAE Val de Loire, 37380 Nouzilly, France

S. Das

Emory Vaccine Center and Department of Pathology and Laboratory Medicine, Emory University, 1462 Clifton Road North-East, Atlanta, GA 30322, USA

P. Pontarotti

SNC5039 CNRS, Paris, France 


\subsection{Preamble}

Eukaryotes and prokaryotes do not have the same DNA content in their genome over stages of their cell differentiation. Indeed genomes are modified during development or cell differentiation. This phenomenon is called developmentally regulated genome rearrangement (DRGR) (Zufall et al. 2005). DRGRs start by a DNA breaks either due to a specific endonuclease activity or specific chromatin structure that is related to a mechanisù inducing a DNA recombination event.

\subsection{Developmentally Regulated Genome Rearrangement via a Specific Endonuclease}

All the reported cases so far correspond to the domestication of selfish DNA that displayed at the minimum an endonuclease activity derived from a DDE transposase, a homing endonuclease, or a prophage recombinase.

The homothallic switching endonuclease (HO) is a domesticated homing endonuclease (Fig. 14.1). The $\mathrm{HO}$ endonucleases are mobile genetic elements that correspond to DNA fragments encoding these proteins over all their length, from their first 5' to the last 3' nucleotide. These DNA fragments use the homing endonuclease they encode to mediate their mobility (Keeling and Roger 1995; Koufopanou and Burt 2005). Homing endonucleases specifically recognize and cleave a specific oligonucleotide motif that is generally located within a very conserved motif of some house-keeping protein-coding sequence. After DNA cleavage, a DNA fragment coding an $\mathrm{HO}$ endonucleases specifically inserts in frame with the parasitized gene in the middle of its own recognition sequences (Fig. 14.1). In a diploid cell that is heterozygous for a homing endonuclease, the gene lacking the parasitic element becomes cleaved at the recognition site, and the broken chromosome is invaded by the parasitic DNA fragment by homologous recombination using the homing endonuclease-containing gene as a template. The mating type in budding yeasts is derived from the domestication of one of these parasitic DNA fragments. The mating types in budding yeasts are encoded by the mating-type (MAT) loci MAT a and MAT $\alpha$. Some yeasts have the ability to change their mating type (mating-type switching) without going through mating or meiosis." In Saccharomyces cerevisiae, switching is initiated when the homothallic switching (HO) endonuclease induces a DNA doublestrand break (DSB) in the MAT locus. Next, the replacement is completed through a gene conversion, in which transcriptionally silent copies of MAT genes, known as "hidden MAT left, $H M L \alpha$ " and "hidden MAT right, HMRa," are copied into the expressed MAT locus.

Prophage excision involved in the specific rearrangement. (Feiner et al. 2015, Fig. 14.2)

During the differentiation process in procaryote, prophages and derived prophages are excised from the genomic region using a prophage recombinase in order to 


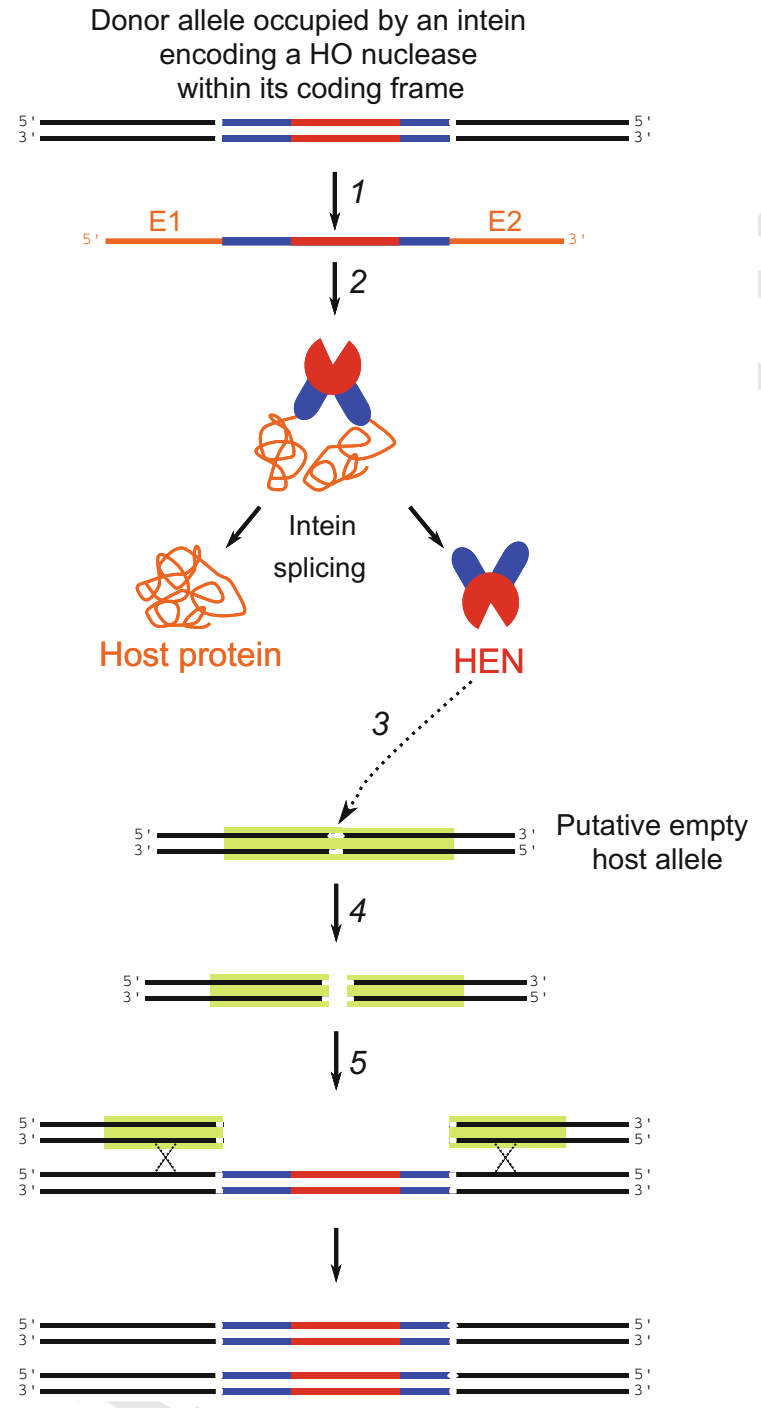

Fig. 14.1 Organization, expression, splicing, and mobility from one allele occupied by an intein to an unoccupied allele. The main steps of the intein mobility and homing are numbered. Step 1 is the RNA transcription. Black lines indicate DNA strands of the occupied and unoccupied alleles by an intein-coding DNA fragment, and cyan blue and light green bars indicate the coding-frame of the DNA region transcribed for each allele kind. Blue and red lines indicated regions coding for the intein (blue) and the homing endonuclease (HEN) moieties (red) in the DNA and the corresponding RNA transcripts. Step 2 is translation into protein. "pacmans" HEN moieties are shown in red and blue ellipses are $\mathrm{N}$ - and $\mathrm{C}$-terminal intein moieties. Step 3 is site-specific recognition of an unoccupied allele by HEN. Blank spaces indicated the insertion site specifically cleaved by the HEN in both alleles. Step 4 is specific cleavage by the HEN. Finally, steps 5 and 6 depicted the invasion of a DNA fragment encoding intein from an occupied allele toward an unoccupied one by DNA strand invasion at DNA replication. This figure was constructed from derived information and graphic elements from Piègu et al. (2015) 
combine ORFs in frame. This process is involved in at least three phenomenons: sporulation (Bacillus and Clostridium), heterocyst differentiation (Cyanobacteria), and monocytogenes phagosomal escape (Listeria).

In the case of the "well analyzed" Bacillus subtilis, gene rearrangement occurs in the sigK gene that is disrupted into two segments by the insertion of SKIN (sigK intervening element) which is a remnant of the ancestral prophage (Abe et al. 2017a). During sporulation, SKIN is excised from the chromosome to combine the ORFs in

\section{a. Lysogenic phage cycle}

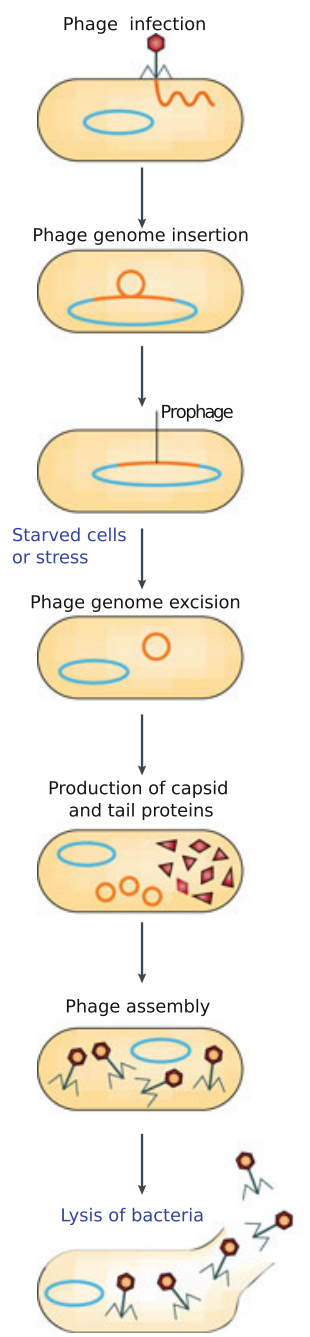

\section{b. Spore development} in Bacillus subtilis

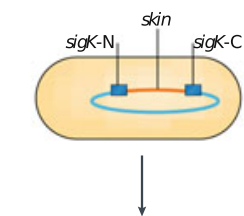

skin regulatory switch excision

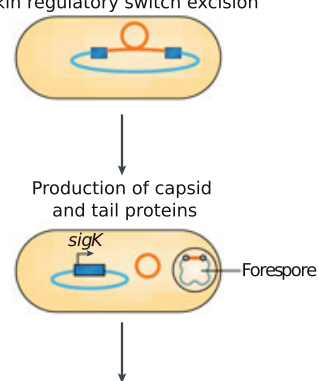

Phage assembly

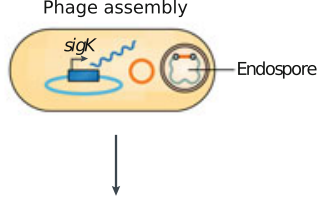

Spore release

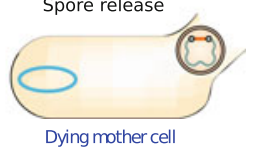


4Fig. 14.2 Parallel between the lysogenic cycle of a phage (a) and the bacterial process involving a phage-derived mechanism activating sporulation $(\mathbf{b})$. a Temperate phages enter a cycle in which their phage genome is first going to be integrated into the bacterial chromosome to become a prophage that is going to persist in what is considered a phage latent or dormant state in which bacterial cells will be viable and will not produce of phage particles. Prophages are replicated together with the bacterial host chromosome during host-cell replication and switch into lytic production upon exposure to stress. Chronologically, the entry in the lytic phase starts with the excision of the phage genome, its gene transcription then the production of phagie capsid and tail proteins that are thereafter assembled in phage particles. The last step is the lysis of the bacteria that releases phages and kills the bacteria. b Regulation of mother cell-specific genes during sporulation in Bacillus subtilis. A phage regulatory switch (phageRS), named skin, is inserted within the $\operatorname{sigK}$ gene. SigK encodes a protein, $\sigma \mathrm{K}$, that regulates the expression of late-stage sporulation genes in the mother cell. skin excises itself at the initiation of the sporulation process, leaving an intact sigK gene able to encode a functional $\sigma \mathrm{K}$ protein. Post excision, $\sigma \mathrm{K}$ expression activates the mother cell's late-stage sporulation genes. Following excision, the excised skin element is eventually lost in the mother cell, which dies late during sporulation. By contrast, the forespore, which did not undergo element excision, gives rise to an endospore that still encodes the skin element within its sigK gene. This figure was constructed from derived information and graphic elements from

frame. In addition to sigK, many other examples of sporulation-specific gene rearrangement occur, suggesting that this phenomenon is widespread and common in spore-forming bacteria (the intervening sequence can correspond to only a recombinase of prophage origin. The recombinase has two functions: it catalyzes the DNA cleavage at the recombination site and join the DNA molecule ends of the restored protein-coding genes (Abe et al. 2017b) (Fig. 14.2)

Heterocysts in cyanobacteria are specialized cells with a role in nitrogen fixation. They provide nitrogen to vegetative cells. Differentiation in a heterocyst is due the excision of fragments that interrupt three different loci: nitD, fdxN, and hupL that encodes a dinitrogenase alpha subunit, a ferredoxin and an uptake hydrogenase large subunit, respectively. The excised fragments have prophage origins and include at least the recombinase gene (Hilton et al. 2016).

Finally, to promote phagosomal escape, Listeria monocytogenes need to excise a temperate prophage integrated into the comK locus during bacterial phagocytosis in order to activate the comk expression (Feiner et al. 2015)

\subsection{Programmed Break Specified by Chromatin Signals}

\subsubsection{Mating-Type Switching in the Fission Yeast S. pombe}

As in the case of $S$. cerevisiae, the yeast Schizosaccharomyces pombe is able of mating-type switching. This mechanism occurred in an independent manner, the two species that belong to two highly divergent clades of yeast. The $S$. pombe genome contains one active (mat1) and two silenced (mat2 and mat3) mating-type loci that share similarities with the $S$. cerevisiae MAT proteins. However, the mechanism 
of switching is different from that involved in S. cerevisiae. Instead of cleavage mediated by an $\mathrm{HO}$ endonuclease (Fig. 14.2), a fragile chromosomal site consisting of an epigenetic mark at the mat locus in switching-competent cells leads to a dsDNA break during replication (Klar et al. 2014).

\subsubsection{Immunoglobulin Switching}

Immunoglobulin isotype class switching is a mechanism that changes a B cell's production of immunoglobulin from one type to another. It involves an intrachromosomal deletional rearrangement that focuses on the region of 1-10 kb of repetitive switch (S) located upstream of each heavy chain isotype gene. Class switch recombination is induced by a break done by cytidine deaminase AID (AID-APOBEC family) on accessible chromatin which is expressed during the B cell development (Yu and Lieber 2019).

The variable lymphocyte receptors (VLRs) system (Fig. 14.3). In agnathes (cyclostomes), the VLR system involved in adaptive immunity includes a germline VLR that do not code for functional protein but instead encodes the only portion of the amino and carboxyl termini of the mature VLRs. The sequences encoding those portions are separated by non-coding intervening regions. In lymphocytes, the germline VLRs are assembled by somatic DNA rearrangement into a mature VLR that encodes the functional receptor via the insertion of LRR cassette that flank the germline VLR. The germline VLR is broken by the AID-APOBEC enzyme at the intervening sequences between the C-terminal and N-terminal portions of the VLR genes where the chromatine is accessible. Then gene conversion starts thanks to sequence identity between the intervening sequence and sequences surrounding the LRR cassettes (Boehm et al. 2018).

\subsubsection{Immunoglobulin Diversity Driven by Gene Conversion in Birds and Some Mammals}

In birds and some mammals, only one pair of functional $\mathrm{V}$ and $\mathrm{J}$ segments is found for both the Ig light and heavy chain loci. Therefore, the diversity generated by $\mathrm{V}(\mathrm{D}) \mathrm{J}$ recombination is limited. However, several pseudo-V coding segments are found upstream the functional $\mathrm{V}$ segment in genes coding light and heavy chains. These pseudo- $\mathrm{V}$ segments are used as a template for gene conversion to diversify the single functional $\mathrm{V}$ segment. To launch the diversification process, a break done by the Activation-induced deaminase (AID) within the single functional $\mathrm{V}$ segment is required. (see for example Arakawa et al. 2002).

Other DRGRs have been described in the literature (Wang and Davis 2014), and they likely correspond to an infinitesimal number of cases that occur in nature. At the 


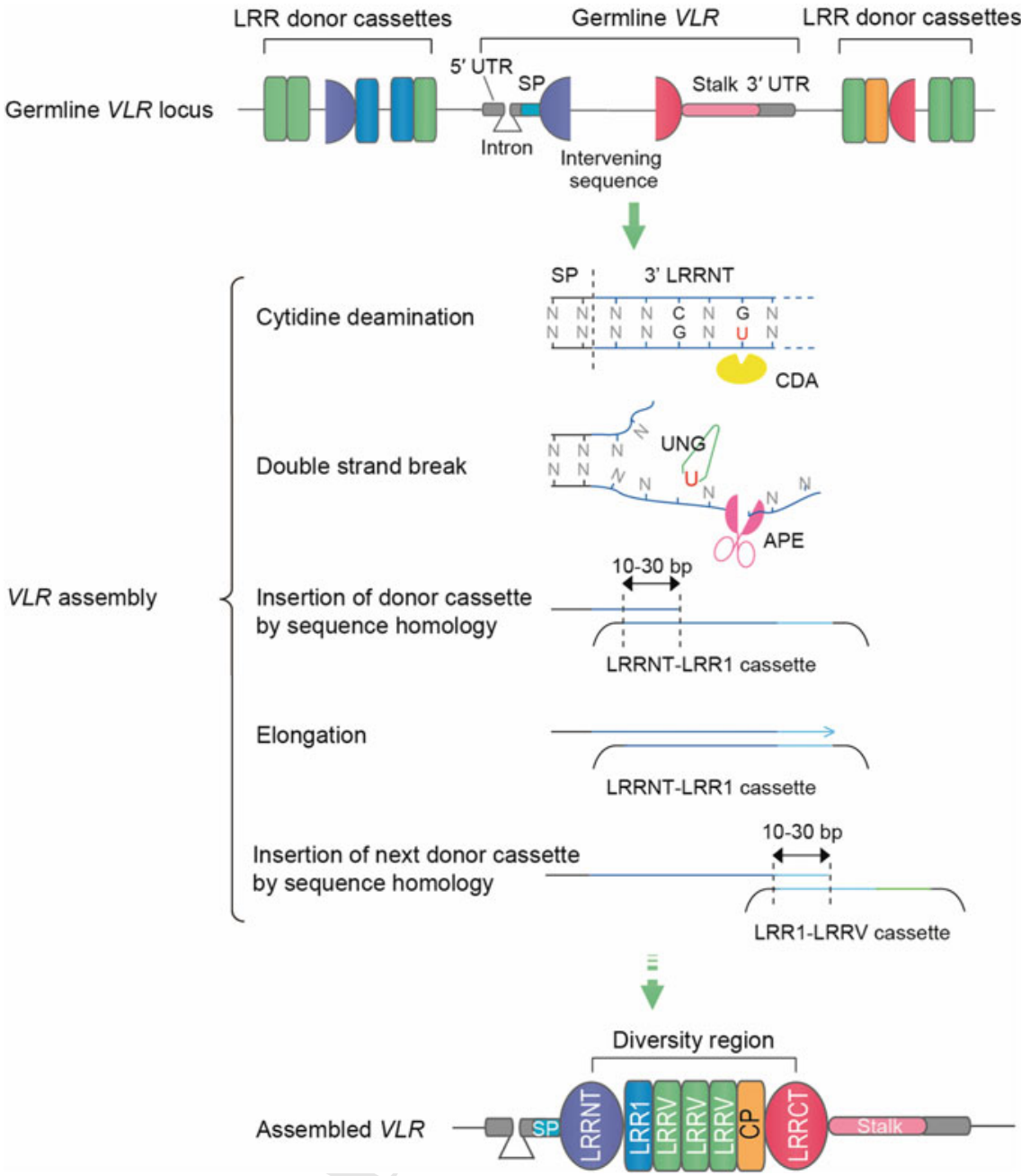

Fig. 14.3 Variable lymphocyte receptors (VLRs) system. Schematic diagram of a germline VLR gene and the postulated gene conversion-like (copy choice) mechanism for $V L R$ assembly. The germline $V L R$ gene is incomplete and contains invariant regions encoding for 5'-end of the Nterminal LRR (5'LRRNT) and $3^{\prime}$-end of the C-terminal LRR (3'LRRCT) and stalk. Hundreds of different LRR cassettes are located upstream and downstream of a pre-assembled germline $V L R$ gene. The non-coding intervening sequence between $5^{\prime} \mathrm{LRRNT}$ and $3^{\prime} \mathrm{LRRCT}$ is replaced by the donor LRR cassettes that are sequentially copied either from $5^{\prime}$ to $3^{\prime}$ or $3^{\prime}$ to $5^{\prime}$ direction. At the beginning of the gene assembly process, a cytosine deaminase (CDA) converts cytosine (C) to uracil $(\mathrm{U})$ in the germline $V L R$ gene. The uracil is then removed by uracil-DNA glycosylase (UNG), leaving an apurinic (AP) site. The AP site activates nicking activity of AP endonuclease (APE) which leads to a DNA double-strand break. To repair this break, homologous recombination starts based on the sequence homology of 10-30 bp between the donor and acceptor LRR cassettes. This process is repeated along with deletion of the intervening sequence until the completion of a mature $V L R$ gene. The diversity region of a mature $V L R$ is composed of a 3'LRRNT, LRR1, multiple LRRVs, connecting peptide (CP), and a 5'LRRCT 
molecular level, as will be seen in the next section beside the prophage and among other domesticated selfish elements, domesticated DDE transposons seem a solution often retained by evolution to achieve programmed DNA elimination.

\subsection{DDE Transposon and Domesticated DDE Transposon}

DNA transposition is the process by which a discrete segment of DNA is either moved or copied into a new genomic location. Several distinct types of enzymes catalyze DNA transposition, one of the most abundant kinds are the DDE transposases thus named for conserved essential acidic residues located at the active catalytic site. The DDE transposase coding gene in the transposon is flanked by two Terminal Inverted Repeats (TIRs). To achieve transposition, the transposase recognizes these TIRs to perform the excision of the transposon which is after this or in a concerted manner with excision is re-inserted into a new genomic location. Upon insertion, the target site DNA is duplicated, resulting in Target Site Duplications (TSDs).

Several DDE transposons have been domesticated by their hosts retaining their DNA binding capacity (see for review (Sinzelle et al. 2009; Jangam et al. 2017)) and in some cases, the transposase and their related TIR as site-specific recombination activating gene. The few well-studied examples will be presented in the next sections.

\subsubsection{Shift from a DDE Transposon to a Mechanism of Programmed DNA Elimination}

DDE transposons have been recruited as site-specific recombination activating gene at least four times as programmed DNA elimination system, mediating biological differentiation processes: RAG (RAG1-RAG2) in jawed vertebrates, Kat 1 and Alpha 3 in K. lactis yeast and PiggyBac (TPB1 TPB2 and TPB6) in the ciliate Tetrahymena. The first described and best known example is the V(D)J RAG.

\subsubsection{The Recombination Activating Gene (RAG) Paradigm}

Jawed vertebrates has a specific adaptive immune system based on lymphocytes that express highly diverse, clonally distributed antigen receptors encoded by genes that are non-functional in the germline and assembled by recombination during lymphocyte development (Teng and Schatz 2015). This assembly reaction, known as $\mathrm{V}(\mathrm{D}) \mathrm{J}$ recombination, operates on arrays of $\mathrm{V}, \mathrm{D}, \mathrm{J}$ polypeptide-coding segments of immunoglobulin and T-cell receptor loci. V(D)J recombination is initiated early in lymphocyte development by a site-specific endonuclease: RAG1 and 2. The RAGs 
cleave at a conserved recombination signal (RSS) that flanks each V D and J segments. The finding that the RAGs have transposase activity, supports a model in which cooption of the components of a transposon played a critical role in the evolution of the jawed vertebrate adaptive immune system (see for review Flajnik 2016) (Fig. 14.4).

The hypothesis was that the RAG proteins derive from transposase genes of RAG transposons while the split antigen receptor genes derive from the insertion of the terminal inverted repeats (TIRs) of this transposon into a Ig-like receptor gene exons with the inserted TIRs becoming the RSSs. The presence of a RAG transposase core and TIRs in non-vertebrates (Kapitonov and Jurka 2005) was consistent with this model. The discovery of a complete RAG transposon in amphioxus (Branchiostoma belcheri) (Huang et al. 2016) strengthens this hypothesis. The discovery of an active

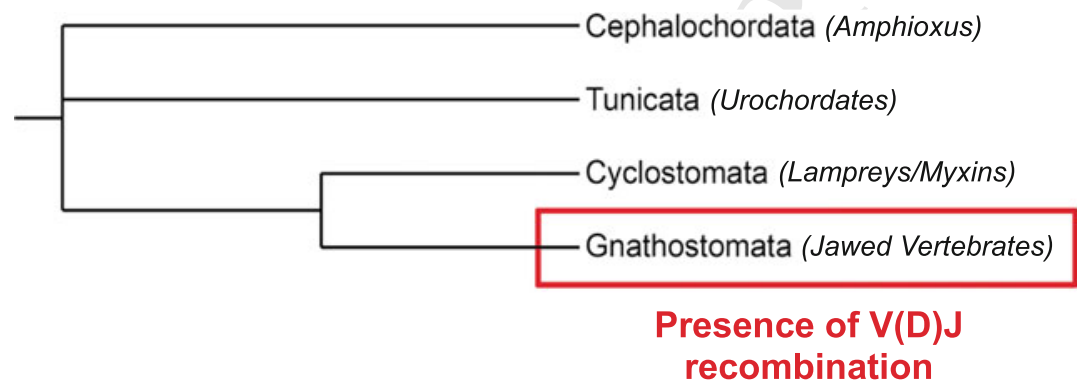

Germline configuration
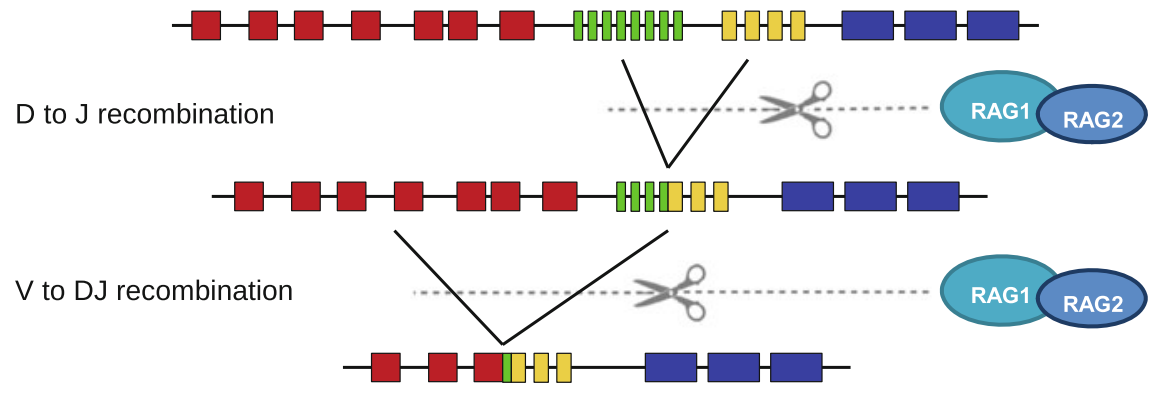

Transcription, splicing
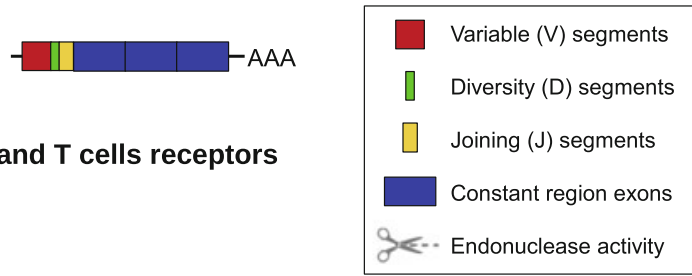

Translation, assembly $\longrightarrow$ B and T cells receptors

Fig. 14.4 RAG implication in the V(D)J recombination mechanism in jawed vertebrates. The chordates consensus tree shows the position of jawed vertebrates among chordates. The V(D)J mechanism was adapted from Janeway et al., 2001 
RAG transposon in the hemichordata Ptychodera flava and several fossilized transposons in several Deuterostomia (Morales Poole et al. 2017) indicate that the RAG transposon was present in the deuterostomia ancestor and remained active to date in several lineages of this clade, while it was co-opted as part of V(D)J recombinase in jawed vertebrates about five hundred millions years ago. The RAG transposon includes TIRs that are related in sequence to the RSS heptamer and the transcribed open reading frame encodes RAG1-like and RAG-2 like proteins with a biochemical activity similar to those of the RAGs, including DNA cleavage via a nick-hairpin mechanism (Huang et al. 2016) (Fig. 14.5).

After DNA cleavage by the jawed vertebrate RAG or Branchiostoma belcheri RAG transposon, there are two predominant fates for the excised fragment flanked by RSSs or TIRs: joining of the ends to form a signal joint or integration into a new locus. The jawed vertebrate recombinase RAG strongly favors the joining of the ends. Indeed, RAG in the jawed vertebrates actively directs cleaved signals and coding ends into the NHEJ DNA repair pathway for joining coding segments in frame. In contrast, Branchiostoma belcheri RAG transposon appears to strongly favor integration after excision, nevertheless, it allows some TIR-TIR joints to form (Huang et al. 2016; Zhang et al. 2019). It is possible that the transposase partially prevents the interaction between the TIR and the NHEJ repair pathway and that the jawed vertebrate RAG lost this property. Zhang et al. (2019) started to uncover the mechanism beyond the domestication and evidenced important amino acid positions involved in transposition/or suppressing transposition (Fig. 14.7).

The mechanism of co-option can be described as follows: (1) insertion of an active transposon into a given genomic locus, (2) the translocation of the transposase gene; in some cases, the native TIRs remain within the genomic locus. (3) The TIR like sequence is recognized by the domesticated transposase (Known under the name of RSSs in the case of RAG) and the TIRs together with the internal sequence are excised (4) the excised sequence lost the ability to insert another genomic region and the two flanking ends are joined by the non-homologous end-joining (NHEJ) DNA repair pathway, to form a coding joint (CJ) (Fig. 14.6).

In conclusion, biochemical functions of the DDE transposon and the sequencespecific recombination activating system are very similar, hence the biochemical shift from a transposon to a sequence-specific recombination activating system corresponds an easy evolutionary step requiring a very limited number of events to pass form a "wild" state to a domesticated one (Fig. 14.3). This is also supported by the fact that many other DDE transposons have been co-opted as recombination activating site-specific endonuclease as described in the next paragraphs.

Piggymac/TPB2/TPB1/TPB6 in ciliates (Baudry et al. 2009; Cheng et al. 2016; Nowacki et al 2009) (Fig. 14.7).

Ciliates are unicellular organisms able to perform DNA rearrangements during development in order to differentiate a somatic macronucleus that is metabolically active from their transcriptionally silent germinal micronucleus. After duplication of the germinal genome, a large proportion of their germinal micronuclear genome is eliminated to differentiate a somatic macronuclear genome through the loss and in particular through the excisions of thousands of internal eliminated sequences. This 

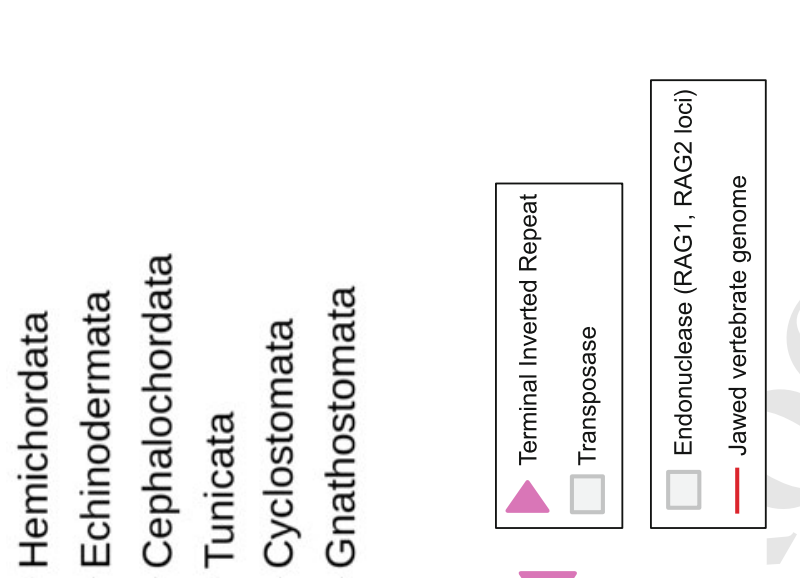

$\frac{\pi}{0} \frac{0}{3}$ $\cong$ 용
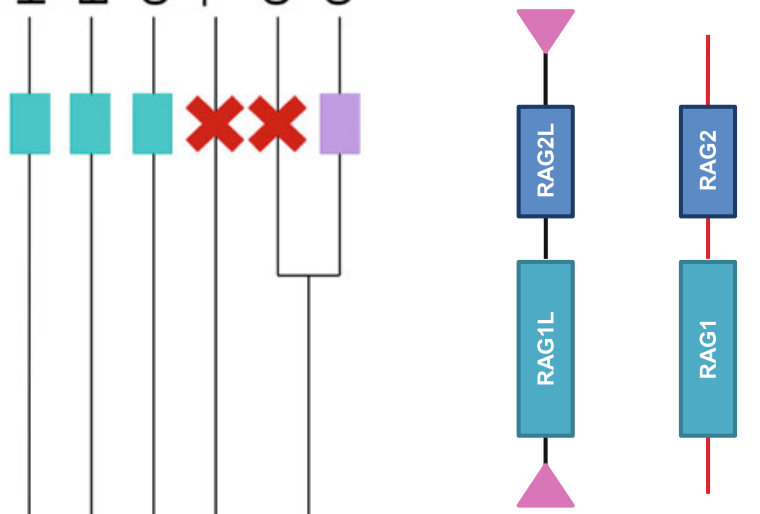

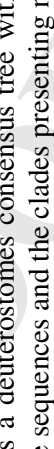

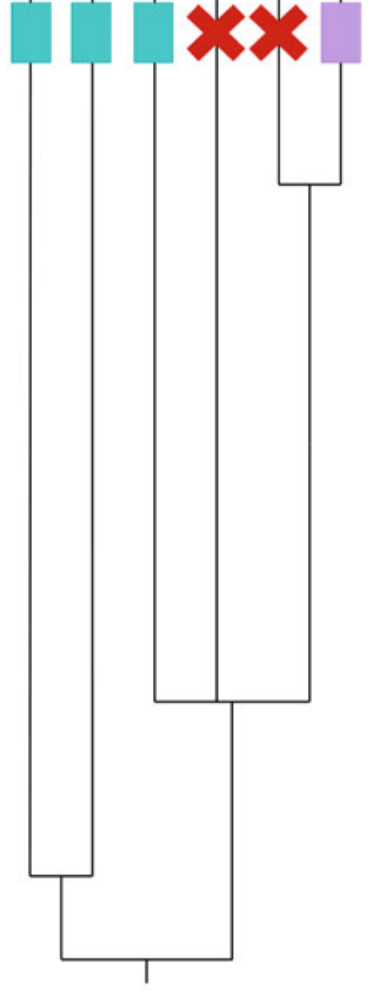

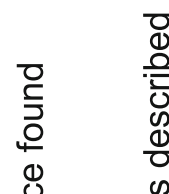

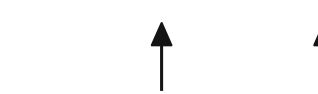

粹

至安

$\dot{\vec{g}} \stackrel{1}{0}$

总.

过

总

.

产

立

造

के

$4 \quad$ 沗

光吉

\&

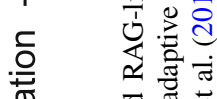

늠

ఝ

บั

の

ㅁํㅇ

๘

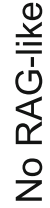

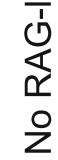

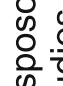

들

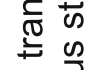

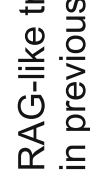

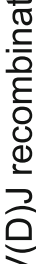

\%

등

ช $\Xi$

둥

巳

낭

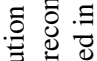

® $\quad$ :

(1)

\)

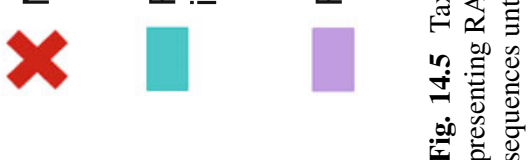

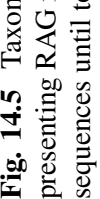

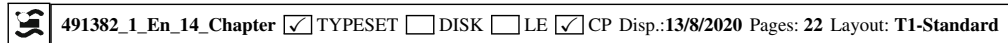



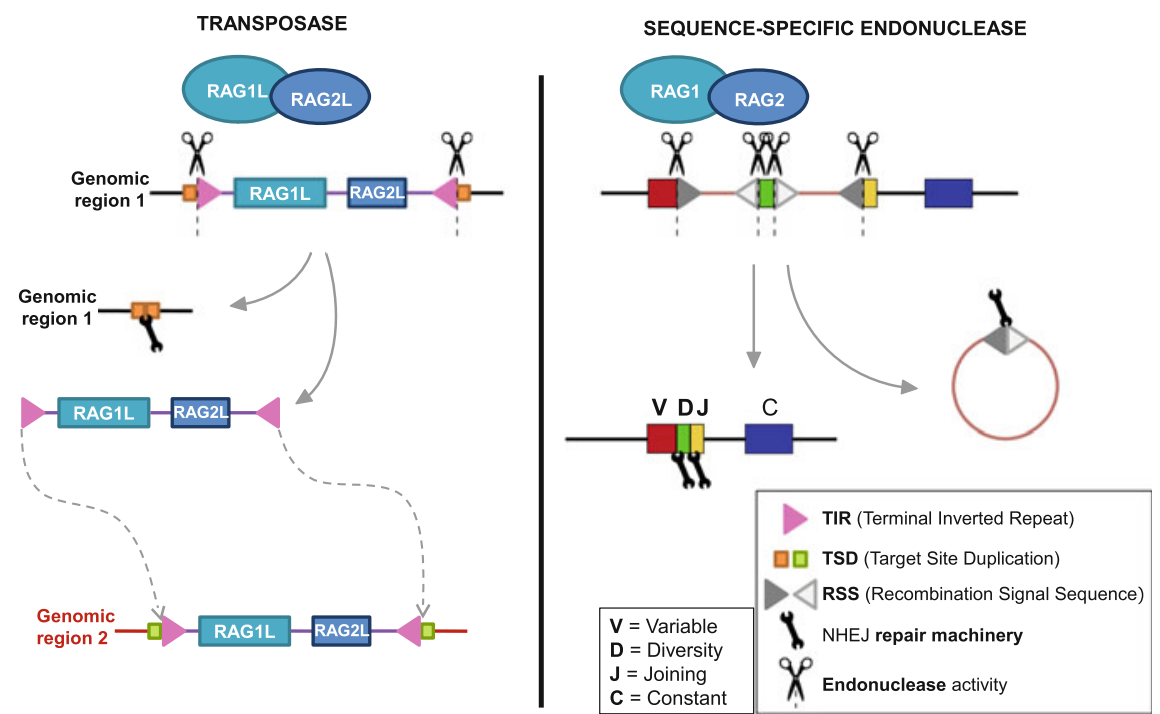

Fig. 14.6 Simple biochemical switch between the RAG-like transposase activity and the RAG endonuclease/recombinase activity in the $\mathrm{V}(\mathrm{D}) \mathrm{J}$ recombination of jawed vertebrates. Adapted from Huang et al. (2016)

is done by domesticated DDE transposon. Most of the eliminated sequences in the paramecium and tetrahymena are named, respectively, by Piggymac and TPB2 and are derived from transposons belonging to the piggybac family (Fig. 14.7). In contrast to the domesticated piggyBac transposase genes in the macronuclear genomes of oligohymenophorea ciliates, Oxytricha bears thousands of active transposase genes within the Tcl/mariner superfamily.

In the case of the Tetrahymena, the domesticated transposons TPB1 and TPB6 excise 12 internal eliminated sequences that disrupt exons. TPB1 and TPB6 recognize the TIR like sequences. TPB2 and likely a Piggymac in paramecium seem to recognize RNA intermediates. In the case of TPB2, the TIR direct interaction with the TIR has been lost and the domesticated transposase performs its excision via scnRNA-directed heterochromatin involved specialization; thus, TPB2 to recognize heterochromatin rather than TIRs. Thus Tetrahymena TPB2 seemingly represents another level of transposase domestication. The situation is less clear in the case of Piggymac where the domesticated transposase seems to recognize both IES ends and the intermediate RNA. It is likely that in the case of TPB2 and Piggymac, the transposition mechanism was gradually grafted into a heterochromatin formation pathway. In all these cases, the internal eliminated sequence form a circle that prevents reintegration in the genome as this is also the case for jawed vertebrates with the chromosomal excision product resulting from the RAG activity.

The phylogenetic analysis (not shown) indicated that possibly a PiggyBac transposon entered the Oligohymenophorea (that include the paramecium and tetrahymena phyla) ancestor and became later domesticated. The ancestor 


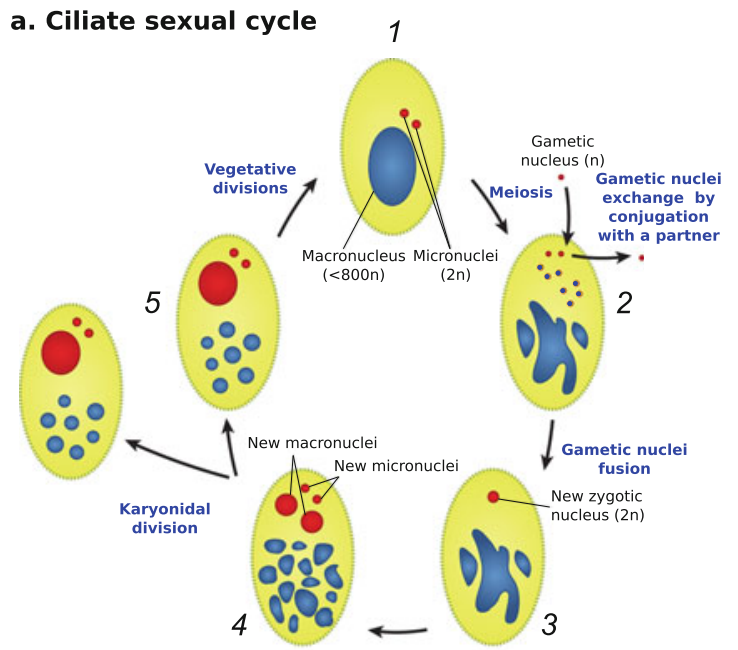

\section{b. Organisation of genome sequences in both nuclei}

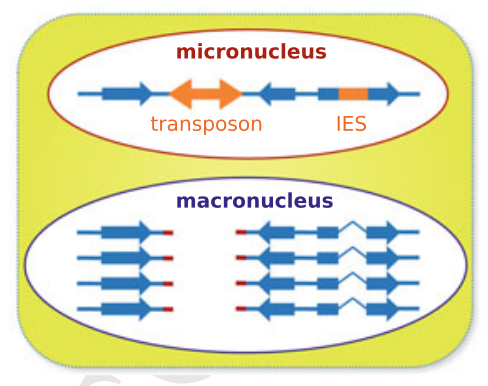

Fig. 14.7 Brief overview of biological and genomic features of the ciliate system. a Main steps of the sexual cycle in ciliate species belonging to the Paramecium genus. At the entry of the cycle in vegetative cells, the macronucleus starts disorganizing while the 2 micronuclei $(2 \mathrm{n})$ trigger meiosis in vegetative cells (1) resulting in eight gametic nuclei (n). Seven or six of these gametic micronuclei, respectively, degenerate after the acquisition of one gametic micronuclei or the exchange of one of them with another Paramecium cell. The fusion of both remaining gametic micronuclei (3) leads to a new zygotic nucleus ( $2 n$ ) that thereafter differentiate in new macronuclei and microneclei (4), before karyonical division and differentiation of a new vegetative cell. b Organization of genome sequences in the micronucleus and the macronucleus. Here, the micronucleus genome is reduced to one chromosome containing the genes (blue arrows) that are for some of them split by insertion element sequence (IES; orange rectangle). DNA transposons (orange double arrows) can also be interspersed in chromosomes. During macronucleus differentiation, IES are precisely excised what restores split gene ORFs (the faint blue line means that the 2 ORF are fusioned in one ORF), transposons are eliminated what fragments chromosomes before the remaining chromosomal fragments are amplified. This figure was constructed from derived information and graphic elements in Nowacki et al. 2011 
Piggymac/TPB2/TPB1/TPB6 domesticated transposon evolved in the two lineages: tetrahymena and paramecium Piggymac and TPB2/TPB1/TPB6. In the case of tetrahymena, a duplication gave rise to TPB2 in one part and TPB1/TPB6 ancestor in the other. TPB2 lost the possibility to recognize its TIR but gained the possibility to interact with the scan RNA. TPB1/TPB6 represent maybe the ancestral state and TPB2 could represent another state in the domestication process.

Kat 1 and MAT alpha3 (Barsoum et al. 2010; Rajaei et al. 2014)

As we said above, some yeasts have the ability to change their mating type. In Saccharomyces cerevisiae, switching is initiated by homothallic switching (HO) endonuclease. In the related yeast Kluyveromyces lactis $\mathrm{HO}$ has been replaced by two domesticated DDE transposons: MATalpha 3 involved in the switch d MAT $\alpha$ to MATa.Katl involved in the switch de MATa to MAT $\alpha$.

$M A T \alpha 3$ which was domesticated from transposable elements belonging to the Mutator Like Element Family MULEs. Regulated excision of this element results in a double stranded DNA break, (DSB) that stimulates recombination from the genome and initiates mating-type switching from MAT $\alpha$ to MATa. It has to be noted that in the case of this domesticated transposon the TIR-like sequences have been replaced by other sequences which are different on the left and right sides. The left side is a low complexity sequence with a long stretch of $\mathrm{T}$ and $\mathrm{A}$. The right side contains a conserved motif that is conserved in sibling species-species of the Kluyveromyces genus. The other domesticated transposon named Kat 1 evolved from hAT (hobo/Activator/Tam3) transposases and is involved in the switching from MATa to $M A T \alpha$. Kat 1 cleaves the MATa locus at two different positions, resulting in DSBs that stimulate recombination. Kat1 recognizes a TIR like sequence. In both cases, the intervening DNA is joined into a circle.

In conclusion, DDE transposons evolved as site-specific recombination activating genes many times during evolution and therefore this is a case of isoconvergent evolution of site-specific recombination activating genes.

\subsubsection{Simple Evolutionary Shift Can Explain Convergent Evolution}

Isoconvergent evolution, the independent evolution of similar features from the same ancestral state in different evolutionary lineages (Pontarotti and Hue 2016), could be explained in part by natural selection where the new feature gave an advantage to the individual. Isoconvergent evolution could be due to the limited number of evolutionary pathways resulting from developmental and functional constraints on the evolutionary process (Losos 2011)_Functional constraints imposing a finite number of accurate adaptations, a finite number of mechanisms can be used to answer functional problems. Finally, isoconvergent evolution can be explained in part by the ease of transition from ancestral state to a derived state. This last aspect is not really discussed in the literature. 
Losos (2011) pointed out that the wings powering flight in vertebrates have been built in different ways in birds, pterosaurs and bats. In all these cases, the wings represent modified forelimbs. The combination of wings and forelimbs, in theory, would be not very useful in real life. He concluded that this was due to lack of constraints; however, we underline here that besides the constraints, it was easier to modify forelimbs to get wings than to start from nothing. The same reasoning could be applied for the DDE transposon co-option as sequence-specific recombination activating systems and the biochemical shift from a transposase to a sequence-specific recombination activating endonuclease is an easy evolutionary step.

\subsubsection{Transposases Form the Largest Family in the Diverse Genomes of Life}

Transposase-encoding genes are greatly over-represented in sequenced genomes and metagenomes relative to other coding sequences (Aziz et al. 2010). Some of the transposase coding genes could correspond to domesticated transposons, active transposons and maybe to fossilized ones. From this and the chapter developed above, we hypothesize that many DDE transposons have been recruited as recombination activating site-specific endonuclease systems. Many domesticated candidates have already been described in the literature, some of them lost the endonuclease domain butand conserved the DNA binding domain and they could be involved in novel chromatin-modifying complexes (Feschotte 2008) while others are involved in centromere binding, chromosome segregation, meiotic recombination (Sinzelle et al. 2009). Some articles also described large-scale systematic analysis to search for domesticated transposons including DDE transposons (Hoen and Bureau 2015; Bouallègue et al. 2017). In the human genome 26 putative DDE transposases has been described (Arnaoty et al. 2012), among these putative domesticated transposases as far as we know other than the RAGs, only the function of one of them, PBGD5 was really investigated, and its nuclease activity has been shown, however we do not know whether it recognizes specific sequences and if it is really involved in recombination (Henssen et al. 2015, 2016, 2017). Another candidate has been tested for its transposase activity (Majumdar et al. 2013) where the authors show that THAP9 gene encodes an active DDE DNA transposase.

We need to test further the hypothesis that many transposons have been recruited as recombination activating site-specific endonuclease. If our hypothesis is true, many domesticated DDE transposons acting as specific DNA endonucleases should be found throughout the life diversity. 


\subsubsection{Search for Domesticated Transposons Involved in Programmed Recombination. Test of the Hypothesis}

This paragraph is a small guideline. The strategy to search for domesticated transposons involved in programmed recombination can be performed by two complementary approaches: Look for the candidate domesticated transposase and look for a somatically rearranged genomic region.

\subsubsection{Look for the Candidate Transposase Co-opted as Site-Specific Endonuclease Involved in Genomic Recombination}

Different strategies have been developed in particular by Hoen and Bureau (2015) and Bouallègue et al. (2017). The strategy described here was adapted from these publications. We first have to look for the sequence that codes for complete transposase; these can be done by profile search using an alignment with a known transposase (Eddy 2011).

The ORF found needs to be checked for a bona fide catalytic site and the conservation of the DDE motif. Then in order to be sure that the transposase is likely to be active it is necessary to show that the protein evolved under constraint. This can be done by calculating the ratio of non-synonymous to synonymous substitutions $(\mathrm{dN} / \mathrm{dS})$ which is a useful measure of the strength and mode of natural selection acting on protein-coding genes (Jeffares et al. 2015).

The next step is to differentiate between transposase belonging to an active transposon from site-specific recombination activating gene (domesticated transposase). The following criteria must be present in the case of site-specific endonuclease. The domesticated transposase must be in single copy without pseudogene like sequences that could correspond to recent transposition events. The next criteria is based on the fact that the domesticated transposase should be unable to transpose thus the domesticated transposase should remain in the same genomic region in different speciesconserved synteny-(Rascol et al. 2009). The higher the number of divergent species display conserved synteny, the higher is the probability that the transposase has been domesticated. For example, PGBD5 is present in all chordate genomes and belongs to a conserved synteny (Pavelitz et al. 2013) indicating that it is likely to have been domesticated.

The following step is to search the domesticated sequence recognition signal: find RSS/TIR like sequence. One way to search for the sequence recognition signal is to perform Chip-Seq experiment (Park 2009), and the other, that could be complementary to the Chip-Seq experiment is to be guided by sequence data. This could be done by looking for the active transposon which is the most similar to the domesticated transposon and to determine the TIR sequence of the transposon. The TIR sequence should be similar to the site-specific DNA sequence recognized by the domesticated 
transposase, as this is the case for the RAG transposon and the domesticated RAG (see Kapitonov and Jurka 2005; Huang et al. 2016; Morales Poole et al. 2017). The TIR like sequences can then be searched in the genome(s) coming from the cells where the "domesticated" transposase is transcribed. It should be noted that the endonuclease could recognize RNA intermediates as this is the case for TPB2 inciliates where the TIR recognition has been lost and instead a chromatin structure is recognized.

\subsubsection{Look for Somatically Rearranged Genomic Regions}

The next step is to look for rearranged genomic regions, and this can be done in several ways. The first way would be to sequence the genome from the cell or the tissue where the domesticated transposase is expressed (sequence 1) compare this genome with a reference genome (sequence 2) from the same species (same individual) in order to look for the rearranged sequence in sequence 1. Another way is to use high throughput genome translocation sequencing methodology based on the ability of a double-strand break to translocate to a fixed 'bait' double-strand break generated by a nuclease (Hu et al. 2016).

In both cases, the genomic region, where the rearrangement occurred, should be flanked by terminal repeats; these terminal repeats corresponding to the RSS should be compared to the TIR of the active transposon (the most similar one).

Candidate regions using the second methodology have been described by Wei et al. (2016). They described 27 recurrent double-strand breaks that occurred during neural stem/progenitor cell differentiation and they named it as recurrent doublestrand break cluster. The authors hypothesized that the break region will be joined to the distal break region thus potentially leading to new genetic information via for example a novel exon combination that can be generated by recombination between intronic regions at the DNA level via "exon shuffling." (Alt and Schwer 2018) In the case where the double-strand break followed by a recombination occurs via the action of domesticated DDE transposon a possible candidate could be PGBD5. Indeed PGBD5 seems to be expressed in the brain and fetal brain and therefore possibly in the neural stem/progenitor cells (Pavelitz et al. 2013) and PGBD5 is able to induce double double-strand breaks in non-physiologic condition (Henssen et al. 2015, 2017).

\subsubsection{Are There Other Domesticated Transposases that Are Able to Generate Diversity?}

One important question concerns the possibility that some domesticated transposase and their TIRs could create diversity through the rearrangement of distinct tandem 
repeat paralogous gene segments (such as in $\mathrm{V}(\mathrm{D}) \mathrm{J}$ recombination). These processes correspond to an excision between at least 2 sets of paralogous fragments with a combinational joining. In these cases, the paralogous fragments should be flanked by the TIR like sequences located $3^{\prime}$ from each of the first paralogous fragments family and $5^{\prime}$ to each of the second fragment paralogue family. Such genomic rearrangement can be searched. The corresponding transposon can be searched as described above.

\subsection{Conclusion}

We discussed in this chapter the possibility that DDE transposons have been recruited several times as systems involved in programmed DNA elimination and perhaps in the generation of receptor diversity. This is due to the fact that the functional shift is an easy one and that the huge number of transposase domains are present and across the tree of life. Therefore, DDE transposons along with other so-called selfish element encoding nucleases (homing nuclease and prophage) could have been major players in the evolution of biodiversity. The co-option of DDE transposons as the regulatory element is also very important. This has been largely discussed, Justin Goodrich this Issue) (Sinzelle et al. 2009; Jangam et al. 2017). Another important role of DDE transposons is the one in the horizontal gene transfer (HGT). Horizontal transfer of DDE transposons (HTT) has been widely reported in eukaryotes (El Baidouri and Panaud 2015). However, in most cases, the literature discusses HGT and HTT in a non-integrated manner. However, the role of DDE transposons as the carrier of additional antibiotic resistance genes is well known in bacteria (Babakhani and Oloomi 2018), and at least the role of DDE transposon as carrier one case in yeast is also known. (McDonald et al. 2019). It is likely that most of the HGT are driven by transposons, but as most of the studied cases HGT corresponds to ancient events the transposase and the corresponding TIR has been lost so an effort in the analysis of recent HGTs should be done. Therefore, DDE transposons could have been major players in organismal evolution because it helps in genetic exchange between species.

The DDE transposon evolutionary trajectory should be included in the public goods hypothesis for the evolution of life on Earth (McInerney et al. 2011). According to this hypothesis, nucleotide sequences are simply seen as goods, passed from one organism to another through both vertical and horizontal transfer. The interesting things about DDE transposons are that they evolved in vertical and horizontal manner and they should be seen as goods since they increase the possibility of transfer and can be co-opted for example as recombination activating site-specific endonucleases involved in programmed DNA elimination.

Acknowledgements This work was supported by the French Government under the «Investissements d'avenir» (Investments for the Future) program managed by the Agence Nationale de la Recherche (ANR, fr: National Agency for Research), (reference: Méditerranée Infection 10-IAHU-03). 


\section{References}

Abe K, Shimizu SY, Tsuda S, Sato T (2017a) A novel non prophage(-like) gene-intervening element within gerE that is reconstituted during sporulation in Bacillus cereus ATCC10987. Sci Rep 7:11426. https://doi.org/10.1038/s41598-017-11796-8

Abe K, Takamatsu T, Sato T (2017b) Mechanism of bacterial gene rearrangement: SprA-catalyzed precise DNA recombination and its directionality control by $\mathrm{SprB}$ ensure the gene rearrangement and stable expression of spsM during sporulation in Bacillus subtilis. Nucleic Acids Res 45:66696683. https://doi.org/10.1093/nar/gkx466

Alt FW, Schwer B (2018) DNA double-strand breaks as drivers of neural genomic change, function, and disease. DNA Repair (Amst) 71:158-163

Arakawa H, HauschiLd J, Buerstedde JM (2002) Requirement of the activation-induced deaminase (AID) gene for immunoglobulin gene conversion. Science (80-) 295:1301-1306. https://doi.org/ 10.1126/science. 1067308

Arnaoty A, Pitard B, Bateau B, Bigot Y, Lecomte T (2012) Novel approach for the development of mew antibodies directed against transposase-derived proteins encoded by human neogenes. Methods Mol Biol 859:293-305. https://doi.org/10.1007/978-1-61779-603-6_17

Aziz RK, Breitbart M, Edwards RA (2010) Transposases are the most abundant, most ubiquitous genes in nature. Nucleic Acids Res 38:4207-4217. https://doi.org/10.1093/nar/gkq140

Babakhani S, Oloomi M (2018) Transposons: the agents of antibiotic resistance in bacteria. J Basic Microbiol 58:905-917

Barsoum E, Martinez P, Åström SU (2010) $\alpha 3$, a transposable element that promotes host sexual reproduction. Genes Dev 24:33-44. https://doi.org/10.1101/gad.557310

Baudry C, Malinsky S, Restituito M, Kapusta A, Rosa S, Meyer E, Bétermier M (2009) PiggyMac, a domesticated piggyBac transposase involved in programmed genome rearrangements in the ciliate Paramecium tetraurelia. Genes Dev 23:2478-2483. https://doi.org/10.1101/gad.547309

Boehm T, Hirano M, Holland SJ, Das S, Schorpp M, Cooper MD (2018) Evolution of alternative adaptive immune systems in vertebrates. Annu Rev Immunol 36:19-42. https://doi.org/10.1146/ annurev-immunol-042617-053028

Bouallègue M, Rouault JD, Hua-Van A, Makni M, Capy P (2017) Molecular evolution of piggyBac superfamily: from selfishness to domestication. Genome Biol Evol 9:323-339. https://doi.org/ 10.1093/gbe/evw292

Cheng CY, Young JM, Lin CYG, Chao JL, Malik HS, Yao MC (2016) The piggyBac transposonderived genes TPB1 and TPB6 mediate essential transposon-like excision during the developmental rearrangement of key genes in Tetrahymena thermophila. Genes Dev 30:2724-2736. https://doi.org/10.1101/gad.290460.116

Eddy SR (2011) Accelerated profile HMM searches. PLoS Comput Biol. https://doi.org/10.1371/ journal.pcbi.1002195

El Baidouri M, Panaud O (2015) Horizontal transfers and the new model of TE-driven genome evolution in Eukaryotes. In: Evolutionary biology: biodiversification from genotype to phenotype. Springer International Publishing, pp 77-92

Feiner R, Argov T, Rabinovich L, Sigal N, Borovok I, Herskovits AA (2015) A new perspective on lysogeny: prophages as active regulatory switches of bacteria. Nat Rev Microbiol 13:641-650

Feschotte C (2008) Transposable elements and the evolution of regulatory networks. Nat Rev Genet 9:397-405

Flajnik MF (2016) Evidence of G.O.D'.s miracle: unearthing a RAG transposon. Cell 166:11-12

Henssen AG, Henaff E, Jiang E, Eisenberg AR, Carson JR, Villasante CM, Ray M, Still E, Burns M, Gandara J, Feschotte C, Mason CE, Kentsis A (2015) Genomic DNA transposition induced by human PGBD5. Elife. https://doi.org/10.7554/elife.10565

Henssen AG, Jiang E, Zhuang J, Pinello L, Socci ND, Koche R, Gonen M, Villasante CM, Armstrong SA, Bauer DE, Weng Z, Kentsis A (2016) Forward genetic screen of human transposase genomic rearrangements. BMC Genom 17:548. https://doi.org/10.1186/s12864-016-2877-x 
Henssen AG, Koche R, Zhuang J, Jiang E, Reed C, Eisenberg A, Still E, Macarthur IC, RodríguezFos E, Gonzalez S, Puiggròs M, Blackford AN, Mason CE, De Stanchina E, Gönen M, Emde AK, Shah M, Arora K, Reeves C, Socci ND, Perlman E, Antonescu CR, Roberts CWM, Steen H, Mullen E, Jackson SP, Torrents D, Weng Z, Armstrong SA, Kentsis A (2017) PGBD5 promotes site-specific oncogenic mutations in human tumors. Nat Genet 49:1005-1014. https://doi.org/10. 1038/ng.3866

Hilton JA, Meeks JC, Zehr JP (2016) Surveying DNA elements within functional genes of heterocyst-forming cyanobacteria. PLoS One. https://doi.org/10.1371/journal.pone.0156034

Hoen DR, Bureau TE (2015) Discovery of novel genes derived from transposable elements using integrative genomic analysis. Mol Biol Evol 32:1487-1506. https://doi.org/10.1093/molbev/ msv042

Hu J, Meyers RM, Dong J, Panchakshari RA, Alt FW, Frock RL (2016) Detecting DNA doublestranded breaks in mammalian genomes by linear amplification-mediated high-throughput genome-wide translocation sequencing. Nat Protoc 11:853-871. https://doi.org/10.1038/nprot. 2016.043

Huang S, Tao X, Yuan S, Zhang Y, Li P, Beilinson HA, Zhang Y, Yu W, Pontarotti P, Escriva H, Le Petillon Y, Liu X, Chen S, Schatz DG, Xu A (2016) Discovery of an active RAG transposon illuminates the origins of V(D)J recombination. Cell 166:102-114. https://doi.org/10.1016/j.cell. 2016.05.032

Jangam D, Feschotte C, Betrán E (2017) Transposable element domestication as an adaptation to evolutionary conflicts. Trends Genet 33:817-831

Jeffares DC, Tomiczek B, Sojo V, dos Reis M (2015) A beginners guide to estimating the nonsynonymous to synonymous rate ratio of all protein-coding genes in a genome. Methods Mol Biol 1201:65-90. https://doi.org/10.1007/978-1-4939-1438-8_4

Kapitonov VV, Jurka J (2005) RAG1 core and V(D)J recombination signal sequences were derived from Transib transposons. PLoS Biol 3:0998-1011. https://doi.org/10.1371/journal.pbio.003 0181

Keeling PJ, Roger AJ (1995) The selfish pursuit of sex. Nature 375:283

Klar AJS, Ishikawa K, Moore S (2014) A unique DNA recombination mechanism of the mating/celltype switching of fission yeasts: a review. Microbiol Spectr. https://doi.org/10.1128/microbiol spec.mdna3-0003-2014

Koufopanou V, Burt A (2005) Degeneration and domestication of a selfish gene in yeast: molecular evolution versus site-directed mutagenesis. Mol Biol Evol 22:1535-1538. https://doi.org/10.1093/ molbev/msi149

Losos JB (2011) Convergence, adaptation, and constraint. Evolution (N Y) 65:1827-1840. https:// doi.org/10.1111/j.1558-5646.2011.01289.x

Majumdar S, Singh A, Rio DC (2013) The human THAP9 gene encodes an active P-element DNA transposase. Science (80-) 339:446-448. https://doi.org/10.1126/science.1231789

McDonald MC, Taranto AP, Hill E, Schwessinger B, Liu Z, Simpfendorfer S, Milgate A, Solomon PS (2019) Transposon-mediated horizontal transfer of the host-specific virulence protein ToxA between three fungal wheat pathogens. MBio. https://doi.org/10.1128/mbio.01515-19

McInerney JO, Pisani D, Bapteste E, O’Connell MJ (2011) The public goods hypothesis for the evolution of life on Earth. Biol Direct 6:41. https://doi.org/10.1186/1745-6150-6-41

Morales Poole JR, Huang SF, Xu A, Bayet J, Pontarotti P (2017) The RAG transposon is active through the deuterostome evolution and domesticated in jawed vertebrates. Immunogenetics 69:391-400. https://doi.org/10.1007/s00251-017-0979-5

Nowacki M, Higgins BP, Maquilan GM, Swart EC, Doak TG, Landweber LF (2009) A functional role for transposases in a large eukaryotic genome. Science 324:935-938

Park PJ (2009) ChIP-seq: advantages and challenges of a maturing technology. Nat Rev Genet 10:669-680

Pavelitz T, Gray LT, Padilla SL, Bailey AD, Weiner AM (2013) PGBD5: a neural-specific introncontaining piggyBac transposase domesticated over 500 million years ago and conserved from cephalochordates to humans. Mob DNA 4:23. https://doi.org/10.1186/1759-8753-4-23 
Pontarotti P, Hue I (2016) Road map to study convergent evolution: A proposition for evolutionary systems biology approaches. Evolutionary biology: convergent evolution, evolution of complex traits, concepts and methods. Springer International Publishing, Heidelberg, pp 3-21

Rajaei N, Chiruvella KK, Lin F, Åström SU (2014) Domesticated transposase Kat1 and its fossil imprints induce sexual differentiation in yeast. Proc Natl Acad Sci U S A 111:15491-15496. https://doi.org/10.1073/pnas.1406027111

Rascol VL, Levasseur A, Chabrol O, Grusea S, Gouret P, Danchin EGJ, Pontarotti P (2009) CASSIOPE: an expert system for conserved regions searches. BMC Bioinf 10:284. https://doi. org/10.1186/1471-2105-10-284

Sinzelle L, Izsvák Z, Ivics Z (2009) Molecular domestication of transposable elements: from detrimental parasites to useful host genes. Cell Mol Life Sci 66:1073-1093

Teng G, Schatz DG (2015) Regulation and evolution of the RAG recombinase. In: Advances in immunology. Academic Press Inc, pp 1-39

Wang J, Davis RE (2014) Programmed DNA elimination in multicellular organisms. Curr Opin Genet Dev 27:26-34

Wei PC, Chang AN, Kao J, Du Z, Meyers RM, Alt FW, Schwer B (2016) Long neural genes harbor recurrent DNA break clusters in neural stem/progenitor cells. Cell 164:644-655. https://doi.org/ 10.1016/j.cell.2015.12.039

Yu K, Lieber MR (2019) Current insights into the mechanism of mammalian immunoglobulin class switch recombination. Crit Rev Biochem Mol Biol 54:333-351

Zhang Y, Cheng TC, Huang G, Lu Q, Surleac MD, Mandell JD, Pontarotti P, Petrescu AJ, Xu A, Xiong Y, Schatz DG (2019) Transposon molecular domestication and the evolution of the RAG recombinase. Nature 569:79-84. https://doi.org/10.1038/s41586-019-1093-7

Zufall RA, Robinson T, Katz LA (2005) Evolution of developmentally regulated genome rearrangements in eukaryotes. J Exp Zool Part B Mol Dev Evol 304:448-455 